\title{
GLOBAL STABILITY OF HOPFIELD NEURAL NETWORKS UNDER DYNAMICAL THRESHOLDS WITH DISTRIBUTED DELAYS
}

\author{
FEI-YU ZHANG AND HAI-FENG HUO
}

Received 14 February 2006; Accepted 25 April 2006

We study the dynamical behavior of a class of Hopfield neural networks with distributed delays under dynamical thresholds. Some new criteria ensuring the existence, uniqueness, and global asymptotic stability of equilibrium point are derived. In the results, we do not require the activation functions to satisfy the Lipschitz condition, and also not to be bounded, differentiable, or monotone nondecreasing. Moreover, the symmetry of the connection matrix is not also necessary. Thus, our results improve some previous works in the literature. These conditions have great importance in designs and applications of the global asymptotic stability for Hopfield neural networks involving distributed delays under dynamical thresholds.

Copyright (c) 2006 F.-Y. Zhang and H.-F. Huo. This is an open access article distributed under the Creative Commons Attribution License, which permits unrestricted use, distribution, and reproduction in any medium, provided the original work is properly cited.

\section{Introduction}

During the last 30 years, Hopfield neural networks (Hopfield [9]) have been extensively studied and developed and have found many applications in different areas such as pattern recognition, model identification, and optimization. Such applications heavily depend on the networks' dynamical behaviors. Therefore, the analysis of dynamical behaviors has important leading significance in the design and application of Hopfield neural networks.

For Hopfield neural networks, one of the most investigated problems in dynamical behaviors is that of the existence, uniqueness, and global asymptotic stability of the equilibrium point. The property of global asymptotic stability, which means that the domain of attraction of the equilibrium point is the whole space and many pseudostable points will be eliminated, is of importance from the theoretical point of view as well as in practical applications in several fields. In particular, globally asymptotically stable Hopfield neural networks were well studied for solving some classes of optimization problems and adaptive control. A globally asymptotically stable Hopfield neural network is guaranteed

Hindawi Publishing Corporation

Discrete Dynamics in Nature and Society

Volume 2006, Article ID 27941, Pages 1-11

DOI 10.1155/DDNS/2006/27941 
to compute the global optimal solution independent of the initial condition and avoid some spurious suboptimal response (Kennedy and Chua [14]; Michel and Gray [17]). Such globally asymptotically stable Hopfield neural networks can also be applied to solve model identification or computational tasks, and so on (Kelly [13]). Thus many scientific and technical workers have been joining the study fields with great interest, and many results about global asymptotic stability of Hopfield neural networks with constant delays and continuous varying delays with boundedness or without delays have been reported (Hopfield [10]; Cao[1]; Zhang and Jin [20]; Marcus and Westervelt [16]; Mohamad [18]; Hirsch [8]; Hopfield and Tank [11]; Liu and Dickson [15]; Huang and Cao [12]; Guan and Chen [7]; Chen [2]). It is well known that the use of constant fixed delays and continuous varying delays provides a good approximation in some simple circuits. However, due to the presence of parallel pathways with a verity of axon sizes and lengths, neural networks usually have a spatial extent and there will be a distribution of conduction velocities along these pathways and a distribution of propagation delays. Under these circumstances, the signal propagation is not instantaneous and cannot be described with discrete delays. An appropriate way is to introduce continuously distributed delay determined by a delay kernel. To the best of our knowledge, few authors have studied the global asymptotic stability of Hopfield neural networks with distributed delays, for example, Feng and Plamondon [4], Gopalsamy and He [5], Zhang and Jin [20], and so on.

On the other hand, Hopfield neural networks with dynamical thresholds have not received any attention until the nineties of the twentieth century. However, Gopalsmy and Leung [6] first considered the Hopfield neural networks with distributed delays under dynamical thresholds as follows:

$$
x^{\prime}(t)=-x(t)+a \tanh \left[x(t)-b \int_{0}^{\infty} k(s) x(t-s) d s-c\right], \quad t \geq 0,
$$

where $x(t) \in C(\mathbb{R}, \mathbb{R})$ is the state of neuron, $k(s) \in C\left(\mathbb{R}^{+}, \mathbb{R}^{+}\right)$is delayed ker-function and satisfies

$$
\begin{gathered}
\int_{0}^{\infty} k(s) d s=1, \\
\int_{0}^{\infty} s k(s) d s<+\infty .
\end{gathered}
$$

$a, b$, and $c$ are nonnegative constants. For their physical meaning of signs in (1.1), one can refer to Gopalsmy and Leung [6]. By using Lyapunov function, they established a sufficient condition ensuring global asymptotic stability of the unique equilibrium point $x^{*}=0$ of system (1.1) with the case $c=0$.

In [22] Zhang et al. consider the more general model as follows:

$$
x^{\prime}(t)=-x(t)+a f\left[x(t)-b \int_{0}^{\infty} k(s) x(t-s) d s-c\right], \quad t \geq 0
$$

where $f: \mathbb{R} \rightarrow \mathbb{R}$ is a globally Lipschitz function. By using Brouwer's theorem and Lyapunov function, they established some sufficient conditions for global asymptotic stability and global exponential stability of equilibrium point $x^{*}$ for the cases $c=0$ and $c \neq 0$. 
Further, Zhang and Li [21] and Zhang et al. [22], respectively, considered the following more general model:

$$
\begin{aligned}
& x_{i}^{\prime}(t)=-d_{i} x_{i}(t)+\sum_{j=1}^{n} a_{i j} f_{j}\left[x_{j}(t)-b_{j} \int_{0}^{\infty} k_{j}(s) x_{j}(t-s) d s-c_{j}\right], \quad t \geq 0, \\
& x_{i}^{\prime}(t)=-g_{i}\left(x_{i}(t)\right)+\sum_{j=1}^{n} a_{i j} f_{j}\left[x_{j}(t)-b_{i j} \int_{0}^{\infty} k_{i j}(s) x_{j}(t-s) d s-c_{j}\right], \quad t \geq 0,
\end{aligned}
$$

where $i=1, \ldots, n . f_{j}: \mathbb{R} \rightarrow \mathbb{R}$ is a global Lipschitz function. By using homeomorphism map in [21] and topological degrees tool in [22], constructing suitable Lyapunov functions, and applying the property of $M$-matrix, they obtained some conditions for the existence, uniqueness, and global asymptotic stability or global exponential stability for model (1.5). However, in all the above-cited literatures, the authors always assumed that the distributed delays $k(s), k_{j}(s)$, or $k_{i j}(s)$ satisfy the property (1.3). In [3] Cui considered the model (1.1) further. By using differential inequality and variations of constants, he dropped the condition (1.3) and obtained new criteria for global asymptotic stability of the equilibrium point $x^{*}=0$ of the system (1.1) with the case $c=0$. However, the method that he used cannot be used for the case $c \neq 0$. In [23] Zhao considered the following model:

$$
x_{i}^{\prime}(t)=-a_{i} x_{i}(t)+\sum_{j=1}^{n} a_{i j} g_{j}\left(x_{j}(t)\right)+b_{i j} \int_{-\infty}^{t} k_{i j}(t-s) g_{j}\left(x_{j}(s)\right) d s+I_{i}, \quad t \geq 0 .
$$

He obtained some condition for global asymptotic stability by dropping the Lipschitz condition and the condition (1.3).

Motivated by the above discussion, our aim in this paper is to study further the existence, uniqueness, and global asymptotic stability for the equilibrium point of the following Hopfield neural network (1.7) with distributed delays under dynamical thresholds:

$$
x_{i}^{\prime}(t)=-d_{i} x_{i}(t)+\sum_{j=1}^{n} a_{i j} f_{j}\left[x_{j}(t)-b_{i j} \int_{0}^{\infty} k_{i j}(s) x_{j}(t-s) d s-c_{j}\right], \quad t \geq 0,
$$

where $i=1,2, \ldots, n, n$ denotes the numbers of units in the neural networks (1.7), $x_{i}(t)$ represents the states of the $i$ th neuron at time $t, a_{i j}, b_{i j}, c_{j}$, and $d_{j}$ are constants, $a_{i j} \geq 0$ denotes the strength of the $j$ th neuron on the $i$ th neuron, $b_{i j} \geq 0$ denotes a measure of the inhibitory influence of the past history of the $j$ th neuron on the ith neuron, $c_{j} \geq 0$ denotes the neural threshold of the $j$ th neuron, and $d_{j}>0$ denotes the rate with which the $j$ th neuron will rest its potential to the resting state in isolation when disconnected from the networks and external inputs. $k_{i j}:[0,+\infty) \rightarrow[0,+\infty)$ is a continuous delayed ker-function satisfying (1.2); $f_{j}$ denotes the output of the $i$ th neuron at time $t$.

The initial condition associated with (1.7) is of the form

$$
x_{0_{i}}(t)=\phi_{i}(t), \quad t \in(-\infty, 0], i=1,2, \ldots, n,
$$


where $\phi_{i} \in C((-\infty, 0], \mathbb{R}), \phi_{i}(t)$ is bounded on $(-\infty, 0]$, and the norm of $C\left((-\infty, 0], \mathbb{R}^{n}\right)$ is denoted by

$$
\|\phi(t)\|=\sup _{t \in(-\infty, 0]} \sum_{i=1}^{n}\left|\phi_{i}(t)\right|
$$

where $\phi(t)=\left(\phi_{1}(t), \ldots, \phi_{n}(t)\right)$. In the results, we assume that the activation functions $f_{j}(j=1, \ldots, n)$ are not the globally Lipschitz functions and the delayed ker-functions $k_{i j}(i, j=1, \ldots, n)$ do not satisfy the assumption (1.3). Here, we point out that our methods are different from differential inequality methods which appeared in Cui [3]. Moreover, our conditions are easy to verify and apply in application fields and the design of Hopfield neural networks.

For convenience, we introduce some notations. Let $x=\left(x_{1}, \ldots, x_{n}\right)^{T}$ denote a column vector (the symbol " $T$ " denotes transpose of $x$ ), let $|x|$ denote the absolute-value vector given by $|x|=\left(\left|x_{1}\right|, \ldots,\left|x_{n}\right|\right)^{T}$, and let $\|x\|=\sum_{i=1}^{n}\left|x_{i}\right|$ denote the norm of $\mathbb{R}^{n}$. For $x(t) \in$ $C\left(\mathbb{R}, \mathbb{R}^{n}\right),\|x(t)\|_{\infty}=\left(\left\|x_{1}(t)\right\|_{\infty}, \ldots,\left\|x_{n}(t)\right\|_{\infty}\right)^{T}$, where $\left\|x_{i}(t)\right\|_{\infty}=\sup _{-\infty<s \leq 0}\left|x_{i}(t+s)\right|$, $i=1, \ldots, n$. For matrices $A$ and $B, A \geq B(A<B)$ means that each pair of corresponding elements of $A \geq B$ satisfies " $\geq$ " (" $<$ "). Especially, $A$ is called a nonnegative matrix if $A \geq 0$.

\section{Existence and uniqueness of the equilibrium point}

In this section, we will study the existence and uniqueness of the equilibrium point of the model (1.7). Before starting our main results, we first rewrite model (1.7) as follows:

$$
X^{\prime}(t)=G(X(t))
$$

in which $X(t)=\left(x_{1}(t), \ldots, x_{n}(t)\right)^{T}$ and

$$
G X(t)=\left(h_{1}(t), \ldots, h_{n}(t)\right)^{T},
$$

where

$$
h_{i}(t)=-d_{i} x_{i}(t)+\sum_{j=1}^{n} a_{i j} f_{j}\left[x_{j}(t)-b_{i j} \int_{0}^{\infty} k_{i j}(s) x_{j}(t-s) d s-c_{j}\right], \quad t \geq 0, i=1, \ldots, n .
$$

In order to study the existence and uniqueness of the equilibrium point, we consider the initial value problem associated with the autonomous system (2.1), in which the initial functions are given by (1.8). Let $\Omega$ be an open subset of $\mathbb{R}^{n}$.

Lemma 2.1. Let $G: \Omega \rightarrow \mathbb{R}^{n}$ be continuous and satisfy the following condition: corresponding to any $\eta \in \Omega$ and its neighborhood $U$, there exist a constant $k>0$ and functions $g_{j}$ and $\Phi_{l}$ $(j, l=1, \ldots, n)$, such that

$$
\|G(\xi)-G(\eta)\| \leq k\|\xi-\eta\|+k \sum_{i=1}^{n}\left|\Phi_{l}\left(g_{j}(\xi)\right)-\Phi_{l}\left(g_{j}(\eta)\right)\right|
$$


on $U$, where each $g_{j}: U \rightarrow \mathbb{R}$ is a continuously differentiable function in $\eta$ satisfying the relation

$$
\sum_{j=1}^{n} \frac{\partial g_{i}(\eta)}{\partial \eta_{i}} G_{i}(\eta) \neq 0 \quad \text { on } U
$$

and each $\Phi_{l}: \mathbb{R} \rightarrow \mathbb{R}$ is continuous and of bounded variation on bounded subintervals. Then there exists a unique solution for the initial value problem system (1.8)-(2.2) on any interval containing the initial functions (1.8).

Proof. For $\eta=\left(x_{1}, \ldots, x_{n}\right)^{T}$, consider the functions $\Phi_{l}(l=1, \ldots, n)$ as follows

$$
\Phi_{l}\left(g_{j}(\eta)\right)=\sum_{j=1}^{n} a_{i j} f_{j}\left[x_{j}(t)-b_{i j} \int_{0}^{\infty} k_{i j}(s) x_{j}(t-s) d s-c_{j}\right],
$$

obviously, for each $l=1, \ldots, n, \Phi_{l}$ is continuous and of bounded variation on bounded subintervals of $\mathbb{R}$. The functions $g_{j}(\eta)$ in (2.6) are also continuously differentiable. So all conditions of [19, Theorem 1], by Norris and Driver hold, and the conclusion of Lemma 2.1. is true.

Obviously, if $x^{*}=\left(x_{1}^{*}, \ldots, x_{n}^{*}\right)$ is an equilibrium point of system (1.7), then $x^{*}$ satisfies

$$
d_{i} x_{i}^{*}=\sum_{j=1}^{n} a_{i j} f_{j}\left[x_{j}^{*}-b_{i j} x_{j}^{*}-c_{j}\right], \quad i=1, \ldots, n .
$$

Theorem 2.2. If the conditions of Lemma 2.1 hold for the functions $f_{j}(j=1, \ldots, n)$, then system (2.7) has a unique solution, therefore, a unique equilibrium point for system (1.7).

Proof. By Lemma 2.1, the system (1.8)-(2.2) has a unique solution $x(t)=\left(x_{1}(t), \ldots\right.$, $\left.x_{n}(t)\right)^{T}$, from this we obtain

$$
\begin{aligned}
x_{i}^{\prime}(t)= & -d_{i}\left(x_{i}(t)-x_{i}^{*}\right) \\
& +\left\{\sum_{j=1}^{n} a_{i j} f_{j}\left[x_{j}(t)-b_{i j} \int_{0}^{\infty} k_{i j}(s) x_{j}(t-s) d s-c_{j}\right]-f_{j}\left[x_{j}^{*}-b_{i j} x_{j}^{*}-c_{j}\right]\right\} .
\end{aligned}
$$

Let $y_{i}(t)=x_{i}(t)-x_{i}^{*}, i=1, \ldots, n$, then it follows from (2.8) that

$$
y_{i}^{\prime}(t)=-d_{i} y_{i}(t)+\sum_{j=1}^{n} a_{i j} g_{j}\left(y_{j}(t)-b_{i j} \int_{0}^{\infty} k_{i j}(s) y_{j}(t-s) d s\right),
$$

where, for $i, j=1, \ldots, n$,

$$
\begin{aligned}
g_{j}\left(y_{j}(t)-b_{i j} \int_{0}^{\infty} k_{i j}(s) y_{j}(t-s) d s\right) \\
\quad=\sum_{j=1}^{n} a_{i j} f_{j}\left[x_{j}(t)-b_{i j} \int_{0}^{\infty} k_{i j}(s) x_{j}(t-s) d s-c_{j}\right]-f_{j}\left[x_{j}^{*}-b_{i j} x_{j}^{*}-c_{j}\right] .
\end{aligned}
$$


The initial condition associated with system (2.9) is of the form

$$
y_{0_{i}}(t)=\varphi_{i}(t)=\phi_{i}(t)-x_{i}^{*}, \quad i=1, \ldots, n \text {. }
$$

Now we choose the initial functions as follows:

$$
y_{i}(t)=0, \quad-\infty<t \leq 0, i=1, \ldots, n .
$$

By Lemma 2.1, the system (2.8) or (2.9) has a unique solution. Obviously, $y_{i}(t)=0$ is the only solution of the system (2.8) or (2.9), which implies that $x_{i}(t)=x_{i}^{*}(i=1, \ldots, n)$ is the unique solution that satisfies (2.8), so there exists a unique point which satisfies (2.7) and guarantees the existence of a unique equilibrium point of the system (1.7). This completes the proof.

\section{Global asymptotic stability of the equilibrium point}

In this section, we will consider global asymptotic stability of system (1.7) and establish some new criteria which do not require the signal propagation functions $f_{i}$ to satisfy the Lipschitz condition and the delayed ker-functions $k_{i j}$ to satisfy the assumption (1.3) which turns out that the hypotheses on the parameters of the system are less restrictive. We note that the equilibrium point $x^{*}$ of system (1.7) is globally asymptotically stable if and only if the equilibrium point $y^{*}=0$ of system (2.9) is globally asymptotically stable.

Throughout this paper, we assume that the following two hypotheses are always true.

(H1) The functions $g_{j}(j=1, \ldots, n)$ satisfy $\operatorname{tg}_{j}(t)>0(t \neq 0)$, and there exist positive constants $L_{j}(j=1, \ldots, n)$ such that

$$
g_{j}(t) \leq L_{j} t \quad \forall t \in \mathbb{R} .
$$

(H2) $\rho(M)<1, M=\left(m_{i j}\right)_{n \times n}, m_{i j}=L_{j} a_{i j}\left(1+b_{i j}\right) / d_{i}$, where the symbol $\rho(M)$ denotes the spectral radius of a square matrix $M$.

Theorem 3.1. Assume that (H1) and (H2) hold. Then the equilibrium point $y^{*}=0$ of system (2.9) is uniformly stable.

Proof. For any $\varepsilon>0$, let $P=\left(p_{1}, \ldots, p_{n}\right)=(I-M)^{-1} E \varepsilon$, where $E=(1, \ldots, 1)^{T}$, which implies $\sum_{j=1}^{n} m_{i j} p_{j}+\varepsilon=p_{i},(i=1, \ldots, n)$. We firstly prove that the set $S=\left\{\Psi \in C \mid\|\Psi\|_{\infty} \leq\right.$ $P\}$ is a positive invariant set of system (2.9). In view of condition (H2) and applying sufficient and necessary conditions of $M$-matrix, we know that $(I-M)^{-1} \geq 0$, and so $(I-M)^{-1} E \varepsilon \geq 0$. In the following, we show that

$$
\|\Psi\|_{\infty} \leq P \text { implies }|y(t)| \leq P \text { for any } t \geq 0, \Psi \in C \text {. }
$$

In order to prove (3.2), we only need to prove that

$$
|y(t)| \leq q P \quad \text { for } t \geq 0 \text { and any given } q>1 .
$$


For the sake of contradiction, we suppose that there exist some $i \in\{1, \ldots, n\}$ and $t_{1}>0$ such that

$$
\begin{gathered}
\left|y_{i}\left(t_{1}\right)\right|=q p_{i}, \quad\left|y_{i}(t)\right|<q p_{i} \quad \text { for } t<t_{1}, \\
|y(t)| \leq q P \quad \text { for } 0 \leq t \leq t_{1} .
\end{gathered}
$$

By (H1), we have

$$
\begin{aligned}
\left|y_{i}\left(t_{1}\right)\right| & \leq e^{-d_{i} t_{1}}|| \Psi_{i} \|_{\infty}+\int_{0}^{t_{1}} e^{-d_{i}\left(t_{1}-s\right)} \sum_{j=1}^{n} a_{i j}\left|g_{j}\left(y_{j}(t)-b_{i j} \int_{0}^{\infty} k_{i j}(\theta) y_{j}(t-\theta) d \theta\right)\right| d s \\
& \leq e^{-d_{i} t_{1}}|| \Psi_{i} \|_{\infty}+\int_{0}^{t_{1}} e^{-d_{i}\left(t_{1}-s\right)} \sum_{j=1}^{n} a_{i j} L_{j}\left(\left|y_{j}(t)\right|+b_{i j} \int_{0}^{\infty} k_{i j}(\theta)\left|y_{j}(t-\theta)\right| d \theta\right) d s \\
& \leq e^{-d_{i} t_{1}} q p_{i}+\int_{0}^{t_{1}} e^{-d_{i}\left(t_{1}-s\right)} \sum_{j=1}^{n} a_{i j} L_{j}\left(q p_{j}+b_{i j} \int_{0}^{\infty} k_{i j}(\theta) q p_{j} d \theta\right) d s \\
& =e^{-d_{i} t_{1}} q p_{i}+\int_{0}^{t_{1}} e^{-d_{i}\left(t_{1}-s\right)}\left(\sum_{j=1}^{n} d_{i} m_{i j} q p_{j}\right) d s \\
& =e^{-d_{i} t_{1}} q p_{i}+\left(1-e^{-d_{i} t_{1}}\right) \sum_{j=1}^{n} m_{i j} q p_{j} \\
& =e^{-d_{i} t_{1}}\left(q p_{i}-\sum_{j=1}^{n} m_{i j} q p_{j}\right)+\sum_{j=1}^{n} m_{i j} q p_{j} .
\end{aligned}
$$

Noting that $q>1$ and $\sum_{j=1}^{n} m_{i j} q p_{j}+q \varepsilon=q p_{i}$, we have

$$
\left|y_{i}\left(t_{1}\right)\right| \leq e^{-d_{i} t_{1}} q \varepsilon+\sum_{j=1}^{n} m_{i j} q p_{j}<q \varepsilon+\sum_{j=1}^{n} m_{i j} q p_{j}=q p_{i},
$$

hence we have a contradiction between (3.4) and (3.7), and so (3.3) holds; letting $q \rightarrow 1$, then (3.2) holds. Therefore, set $S$ is a positive invariant set of system (2.9), and it follows that the equilibrium point $y(t)=0$ of system (2.9) is uniformly stable by using the relation between positive invariant set and uniform stability. The proof is complete.

In view of the above proof, we have the following result.

Corollary 3.2. Assume that (H1) and (H2) hold. Then all the solutions of system (2.9) are uniformly bounded.

Proof. By the proof of Theorem 3.1, we have that for any given $\Psi \in C$ there must be $q>1$ such that all the solutions of system (2.9) satisfy $|y(t)|<q p$ for $t \geq 0$ and $\|\Psi\|_{\infty}<q p$, which implies that the conclusion of Corollary 3.2 is true.

Theorem 3.3. Assume that (H1) and (H2) hold. Then the equilibrium point $y^{*}=0$ of system (2.9) is globally attractive. 
Proof. For any given $\Psi \in C$, we only need to prove $\lim _{t \rightarrow \infty} \sup |y(t)|=0$, where $\lim _{t \rightarrow \infty}$ $\sup |y(t)|=\left(\lim _{t \rightarrow \infty} \sup \left|y_{1}(t)\right|, \ldots, \lim _{t \rightarrow \infty} \sup \left|y_{n}(t)\right|\right)$. According Corollary 3.2, there exists a nonnegative constant vector $\sigma=\left(\sigma_{1}, \ldots, \sigma_{n}\right)^{T}$ such that $\lim _{t \rightarrow \infty} \sup |y(t)|=\sigma$, therefore, for sufficient small constant $\varepsilon>0$, there exists $t_{2}>0$, such that

$$
|y(t)| \leq(1+\varepsilon) \sigma \quad \text { for any } t \geq t_{2} .
$$

By Corollary 3.2, there must be a positive constant $Q>0$ such that $|y(t)| \leq Q$ for $t \in \mathbb{R}$ and any given $\Psi \in C$. In view of condition (1.2), for the above $\varepsilon>0$, there must be $T>0$ such that

$$
\int_{0}^{T} k_{i j}(s) d s \leq 1, \quad\left(\sum_{j=1}^{n} a_{i j} L_{j} b_{i j} Q\right) \int_{T}^{\infty} k_{i j}(s) d s<\varepsilon, \quad i=1, \ldots, n .
$$

From (2.9), (3.8), and (3.9), when $t \geq t_{2}+T$,

$$
\begin{aligned}
y_{i}^{\prime}(t)+d_{i} y_{i}(t) & \leq \sum_{j=1}^{n} a_{i j} L_{j}\left(\left|y_{j}(t)\right|+b_{i j} \int_{0}^{\infty} k_{i j}(s)\left|y_{j}(t-s)\right| d s\right) \\
& =\sum_{j=1}^{n} a_{i j} L_{j}\left[\left|y_{j}(t)\right|+b_{i j}\left(\int_{0}^{T} k_{i j}(s)\left|y_{j}(t-s)\right| d s+\int_{T}^{\infty} k_{i j}(s)\left|y_{j}(t-s)\right| d s\right)\right] \\
& \leq \sum_{j=1}^{n} a_{i j} L_{j}\left[(1+\varepsilon) \sigma_{j}+b_{i j} \int_{0}^{T} k_{i j}(s)(1+\varepsilon) \sigma_{j} d s+b_{i j} Q \int_{T}^{\infty} k_{i j}(s) d s\right] \\
& <d_{i}\left(\sum_{j=1}^{n} m_{i j}(1+\varepsilon) \sigma_{j}+\varepsilon\right)
\end{aligned}
$$

hence it follows that

$$
\begin{aligned}
\left|y_{i}(t)\right| & <e^{-d_{i} t}|| \Psi \|_{\infty}+\int_{0}^{t} e^{-d_{i}\left(t_{1}-s\right)} d_{i}\left(\sum_{j=1}^{n} m_{i j}(1+\varepsilon) \sigma_{j}+\varepsilon\right) d s \\
& <e^{-d_{i} t}\|\Psi\|_{\infty}+\left(1-e^{-d_{i} t}\right)\left(\sum_{j=1}^{n} m_{i j}(1+\varepsilon) \sigma_{j}+\varepsilon\right)
\end{aligned}
$$

then letting $t \rightarrow+\infty$, we have $\lim _{t \rightarrow+\infty} \sup \left|y_{i}(t)\right| \leq \sum_{j=1}^{n} m_{i j} \sigma_{j}, i=1, \ldots, n$, thus $\sigma_{i} \leq$ $\sum_{j=1}^{n} m_{i j} \sigma_{j}$, namely, $(I-M) \sigma \leq 0$. Since $(I-M)^{-1} \geq 0$, so $\sigma \leq 0$, note that $\sigma$ is a nonnegative constant. It follows that $\sigma=0$, therefore, $\lim _{t \rightarrow \infty} \sup |y(t)|=0$. This completes the proof.

By Theorems 3.1 and 3.3, we conclude the following result.

Theorem 3.4. Assume that (H1) and (H2) hold. Then the equilibrium point $y^{*}=0$ of system (2.9) is globally asymptotically stable. Therefore, the equilibrium point $x^{*}$ of system (1.7) is globally asymptotically stable.

If the signal propagation functions $g_{j}$ are globally Lipschitz functions, we have the following corollary. 
Corollary 3.5. Assume that the signal propagation functions $f_{j}$ are globally Lipschitz with Lipschitz constants $L_{j}>0$ and condition (H2) holds. Then the equilibrium point $x^{*}$ of system (1.7) is globally asymptotically stable.

Remark 3.6. When $n=1$ and the signal propagation functions $f(t)=\tanh x$, the system (1.7) becomes the system (1.1) which is firstly considered by Gopalsmy and Leung [6]; in their article, they assumed that the delayed ker-function $k(s)$ satisfies the condition (1.3) and obtained global asymptotic stability of the unique equilibrium $x^{*}=0$ under the conditions $a(1-b)<1$ and $a(1+b)<1$ for the case $c=0$. Cui [3] also considered the system (1.1); he deleted the condition (1.3) and obtained global asymptotic stability of the unique equilibrium $x^{*}=0$ under the conditions $a(1-b)<1$ and $a(1+b)<1$ for the case $c=0$. Obviously, in the above-mentioned article, the signal propagation functions $f(t)=\tanh t$ is globally Lipschitz. Zhang et al. [22] considered the system (1.4) which generalizes the system (1.1); they also assumed that the delayed-ker-function $k(s)$ satisfies the condition (1.3) and the signal propagation function $f(t)$ is globally Lipschitz, and obtained global asymptotic stability of the unique equilibrium $x^{*}=0$ under the condition $a(1+b)<d$ for the cases $c=0$ and $c \neq 0$. However, in Theorem 3.4 and Corollary 3.5 of this paper, the signal propagation functions $f_{j}(t)$ are not globally Lipschitz and the condition (1.3) is not also needed. Clearly, our results in this paper contain and improve those given in above-mentioned literatures, and the conditions in this paper require less restrictive parameters than those given also in the above-mentioned articles.

Remark 3.7. Zhang and Li [21] and Zhang et al. [22] considered the system (1.5) which further generalizes the system (1.1). When the signal propagation functions $f_{j}(t)$ are globally Lipschitz with Lipschitz constants $L_{j}$ and condition (1.3) holds, they proved that the unique equilibrium point $x^{*}$ is globally asymptotically stable under the condition $M=D L^{-1}-A(E+B)$ is an $M$-matrix, clearly $M$ is an $M$-matrix is equivalent to the condition $\rho(M)<1$. However, the condition (1.3) and the Lipschitz condition associated to the signal propagation functions $f_{j}(t)$ are not necessary in Theorem 3.4 of this paper; obviously, the results in this paper improve the previous works.

\section{Two illustrative examples}

In this section, we will give two examples to illustrate our results.

Example 4.1. Consider the following model with two neurons:

$$
\begin{aligned}
x_{1}^{\prime}(t)= & -x_{1}(t)+\frac{1}{3} \tanh \left[x_{1}(t)-\frac{1}{5} \int_{0}^{\infty} \frac{2}{\pi\left(1+s^{2}\right)} x_{1}(t-s) d s-1\right] \\
& +\frac{1}{3} \tanh \left[x_{2}(t)-\frac{1}{3} \int_{0}^{\infty} \frac{2}{\pi\left(1+s^{2}\right)} x_{2}(t-s) d s-2\right], \\
x_{2}^{\prime}(t)= & -x_{2}(t)+\frac{1}{3} \tanh \left[x_{1}(t)-\frac{1}{3} \int_{0}^{\infty} \frac{2}{\pi\left(1+s^{2}\right)} x_{1}(t-s) d s-1\right] \\
& +\frac{1}{3} \tanh \left[x_{2}(t)-\frac{1}{3} \int_{0}^{\infty} \frac{2}{\pi\left(1+s^{2}\right)} x_{2}(t-s) d s-2\right] .
\end{aligned}
$$


Obviously, $k_{i j}(s)=2 / \pi\left(1+s^{2}\right)$ satisfies condition (1.2), and functions $f_{j}(t)=\tanh t$ satisfy condition (H1) and also are globally Lipschitz with $L_{j}=1$. It is easy to verify that the matrix $M=\left[\begin{array}{ll}2 / 3 & 4 / 9 \\ 4 / 9 & 4 / 9\end{array}\right]$ and $\rho(M)<1$. However, we should note that $k_{i j}(s)$ does not satisfy the condition (1.3). Therefore, by Theorem 3.4 or Corollary 3.5, system (4.1) has a globally asymptotically stable equilibrium point.

Example 4.2. Consider the following model with two neurons:

$$
\begin{aligned}
x_{1}^{\prime}(t)= & -2 x_{1}(t)+\frac{1}{3} g\left[x_{1}(t)-\frac{1}{4} \int_{0}^{\infty} \frac{2}{\pi\left(1+s^{2}\right)} x_{1}(t-s) d s-1\right] \\
& +\frac{1}{3} g\left[x_{2}(t)-\frac{1}{3} \int_{0}^{\infty} \frac{2}{\pi\left(1+s^{2}\right)} x_{2}(t-s) d s-2\right], \\
x_{2}^{\prime}(t)= & -x_{2}(t)+\frac{1}{4} g\left[x_{1}(t)-\frac{1}{3} \int_{0}^{\infty} \frac{2}{\pi\left(1+s^{2}\right)} x_{1}(t-s) d s-1\right] \\
& +\frac{1}{2} g\left[x_{2}(t)-\frac{1}{3} \int_{0}^{\infty} \frac{2}{\pi\left(1+s^{2}\right)} x_{2}(t-s) d s-2\right],
\end{aligned}
$$

where $g_{j}(s)=\int_{-\infty}^{s} x_{j}(\theta) e^{\theta-s} d \theta$ and $x_{j}(s)$ equals one when a pulse arrives at time $t$ and zero when no pulse arrives. Obviously, $g_{j}(s)$ satisfies condition (H1) with $L_{j}=1$, but it does not have to be a globally Lipschitz function. $k_{i j}(s)=2 / \pi\left(1+s^{2}\right)$ satisfies the condition (1.2) but it does not satisfy the condition (1.3). It is easy to verify that $M=\left[\begin{array}{cc}5 / 24 & 2 / 9 \\ 1 / 3 & 2 / 3\end{array}\right]$ is an $M$-matrix, so the condition (H2) of Theorem 3.4 holds. Therefore, By Theorem 3.4, the system (4.2) has a globally exponentially stable equilibrium.

\section{Acknowledgments}

The second author was supported by the NSF of Gansu Province of China (Grant no. 3ZS042-B25-013), the NSF of Bureau of Education of Gansu Province of China (Grant no. 0416B-08), the Key Research and Development Program for Outstanding Groups of Lanzhou University of Technology, and the Development Program for Outstanding Young Teachers in Lanzhou University of Technology.

\section{References}

[1] J. D. Cao, On exponential stability and periodic solutions of CNNs with delays, Physics Letters. A 267 (2000), no. 5-6, 312-318.

[2] J. P. Chen, Global exponential stability of delayed Hopfield neural networks, Neural Networks 14 (2001), no. 8, 977-980.

[3] W. Y. Cui, Global stability for a class of neural network models under dynamical thresholds with delays, Journal of Biomathematics 15 (2000), no. 4, 420-424 (Chinese).

[4] C. H. Feng and R. Plamondon, On the stability analysis of delayed neural networks systems, Neural Networks 14 (2001), no. 9, 1181-1188.

[5] K. Gopalsamy and X.-Z. He, Delayed-independent stability in bidirectional associative memory networks, IEEE Transactions on Neural Networks 5 (1994), no. 6, 998-1002. 
[6] K. Gopalsmy and I. K. C. Leung, Convergence under dynamical thresholds with delays, IEEE Transactions on Neural Networks 8 (1997), no. 2, 341-348.

[7] Z. H. Guan and G. R. Chen, On delayed impulsive Hopfield neural networks, Neural Networks 12 (1999), no. 2, 273-280.

[8] M. W. Hirsch, Convergent activation dynamics in continuous-time networks, Neural Networks 2 (1989), no. 5, 331-349.

[9] J. J. Hopfield, Neural networks and physical systems with emergent collective computational abilities, Proceedings of the National Academy of Sciences of the United States of America 79 (1982), no. $8,2554-2558$.

[10] _ Neurons with graded response have collective computational properties like those of twostate neurons, Proceedings of the National Academy of Sciences of the United States of America 81 (1984), no. 10, 3088-3092.

[11] J. J. Hopfield and D. W. Tank, "Neural" computation of decisons in optimization problems, Biological Cybernetics 52 (1985), no. 3, 141-152.

[12] H. Huang and J. D. Cao, On global asymptotic stability of recurrent neural networks with timevarying delays, Applied Mathematics and Computation 142 (2003), no. 1, 143-154.

[13] D. G. Kelly, Stability in contractive nonlinear neural networks, IEEE Transactions on Biomedical Engineering 37 (1990), no. 3, 231-242.

[14] M. P. Kennedy and L. O. Chua, Neural networks for nonlinear programming, IEEE Transactions on Circuits and Systems 35 (1988), no. 5, 554-562.

[15] X. Z. Liu and R. Dickson, Stability analysis of Hopfield neural networks with uncertainty, Mathematical and Computer Modelling 34 (2001), no. 3-4, 353-363.

[16] C. M. Marcus and R. M. Westervelt, Stability of analog neural networks with delay, Physical Review. A 39 (1989), no. 1, 347-359.

[17] A. N. Michel and D. L. Gray, Analysis and synthesis of neural networks with lower block triangular interconnecting structure, IEEE Transactions on Circuits and Systems 37 (1990), no. 10, 12671283.

[18] S. Mohamad, Convergence dynamics of delayed Hopfield-type neural networks under almost periodic stimuli, Acta Applicandae Mathematicae 76 (2003), no. 2, 117-135.

[19] M. J. Norris and R. D. Driver, A uniqueness theorem for ordinary differential equations, SIAM Journal on Mathematical Analysis 12 (1981), no. 2, 141-144.

[20] J. Y. Zhang and X. S. Jin, Global stability analysis in delayed Hopfield neural network models, Neural Networks 13 (2000), no. 7, 745-753.

[21] F.-Y. Zhang and W.-T. Li, Global stability of delayed Hopfield neural networks under dynamical thresholds, Discrete Dynamics in Nature and Society 2005 (2005), no. 1, 1-17.

[22] F.-Y. Zhang, W.-T. Li, and H.-F. Huo, Global stability of a class of delayed cellular neural networks with dynamical thresholds, International Journal of Applied Mathematics 13 (2003), no. 4, 359368.

[23] H. Y. Zhao, Global asymptotic stability of Hopfield neural networks involving distributed delays, Neural Networks 17 (2004), no. 1, 47-53.

Fei-Yu Zhang: Department of Mathematics, Hexi University, Zhangye, Gansu 734000, China E-mail address: zhfy@hxu.edu.cn

Hai-Feng Huo: Institute of Applied Mathematics, Lanzhou University of Technology, Lanzhou, Gansu 730050, China

E-mail address: hfhuo@lut.cn 


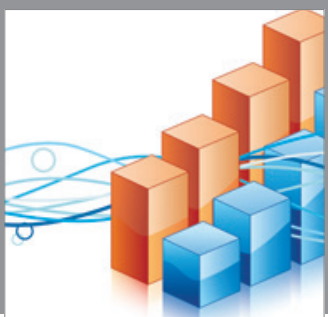

Advances in

Operations Research

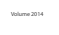

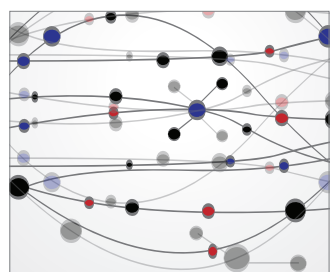

\section{The Scientific} World Journal
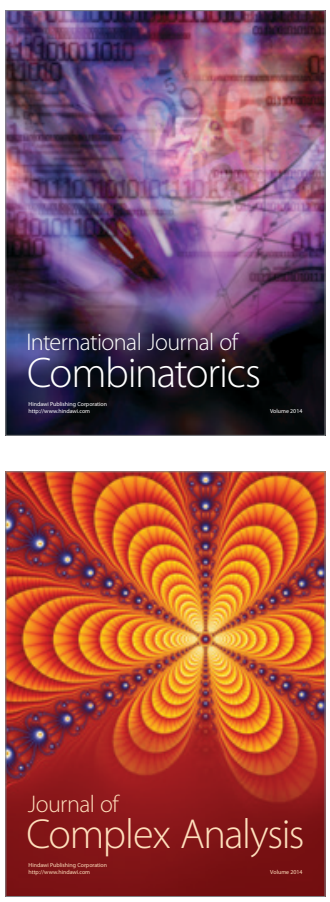

International Journal of

Mathematics and

Mathematical

Sciences
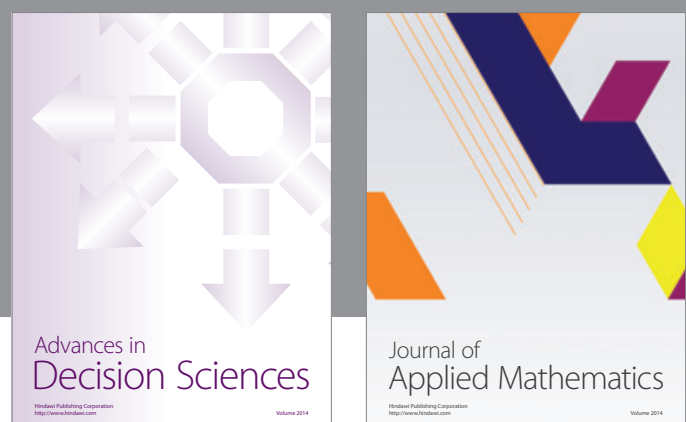

Journal of

Applied Mathematics
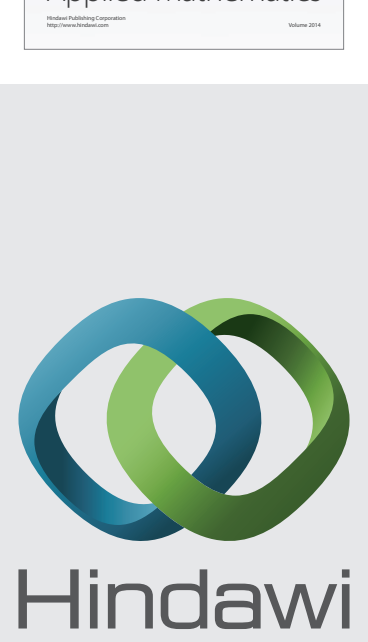

Submit your manuscripts at http://www.hindawi.com


Mathematical Problems in Engineering
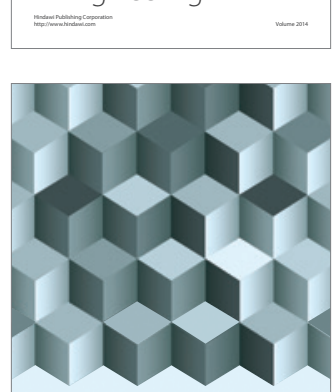

Journal of

Function Spaces
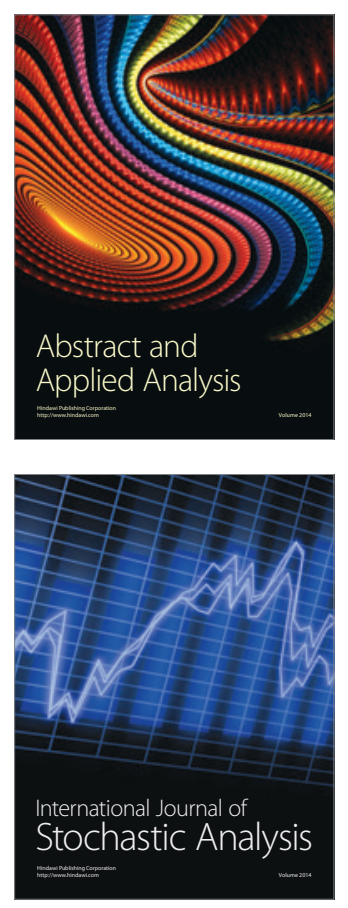



ournal of

Probability and Statistics

Promensencen
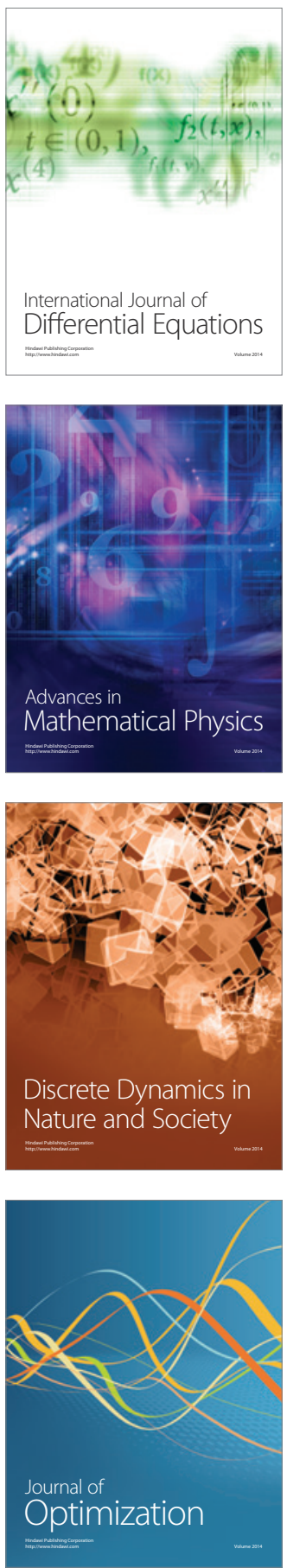